\title{
Microscopic origin of black hole reentrant phase transitions
}

\author{
M. Kord Zangeneh, ${ }^{1,2, *}$ A. Dehyadegari, ${ }^{3, \dagger}$ A. Sheykhi, ${ }^{3,2, *}$ and R. B. Mann ${ }^{4, \S}$ \\ ${ }^{1}$ Physics Department, Faculty of Science, Shahid Chamran University of Ahvaz, Ahvaz 61357-43135, Iran \\ ${ }^{2}$ Research Institute for Astronomy and Astrophysics of Maragha (RIAAM), \\ P.O. Box 55134-441 Maragha, Iran \\ ${ }^{3}$ Physics Department and Biruni Observatory, Shiraz University, Shiraz 71454, Iran \\ ${ }^{4}$ Department of Physics and Astronomy, University of Waterloo, Waterloo, Ontario, Canada N2L $3 G 1$
}

(Received 23 September 2017; published 27 April 2018)

\begin{abstract}
Understanding the microscopic behavior of the black hole ingredients has been one of the important challenges in black hole physics during the past decades. In order to shed some light on the microscopic structure of black holes, in this paper, we explore a recently observed phenomenon for black holes namely reentrant phase transition, by employing the Ruppeiner geometry. Interestingly enough, we observe two properties for the phase behavior of small black holes that leads to reentrant phase transition. They are correlated and they are of the interaction type. For the range of pressure in which the system underlies reentrant phase transition, it transits from the large black holes phase to the small one which possesses higher correlation than the other ranges of pressures. On the other hand, the type of interaction between small black holes near the large/small transition line differs for usual and reentrant phase transitions. Indeed, for the usual case, the dominant interaction is repulsive whereas for the reentrant case we encounter an attractive interaction. We show that in the reentrant phase transition case, the small black holes behave like a bosonic gas whereas in the usual phase transition case, they behave like a quantum anyon gas.
\end{abstract}

DOI: 10.1103/PhysRevD.97.084054

\section{INTRODUCTION}

A reentrant phase transition (RPT) occurs when a monotonic variation of any thermodynamic quantity gives rise to more than one phase transition (PT) such that the initial and final states are macroscopically the same. This phenomenon was first discovered in the nicotine/water mixture during a procedure in which, by increasing the temperature at a sufficient fixed percentage of nicotine, the homogeneous mixed state separated into distinct nicotine/ water phases and then the homogeneous state reappeared [1]. More often, as a result of two (or more) competing driving mechanisms, such behavior has also been observed in multicomponent fluid systems, gels, ferroelectrics, liquid crystals, and binary gases as well as noncommutative spacetimes [2] (for more details, see the review [3]).

In the context of black hole (BH) physics, RPT has been first discovered for four-dimensional Born-Infeld (BI)

\footnotetext{
*mkzangeneh@scu.ac.ir

†adehyadegari@shirazu.ac.ir

asheykhi@ shirazu.ac.ir

rbmann@uwaterloo.ca
}

Published by the American Physical Society under the terms of the Creative Commons Attribution 4.0 International license. Further distribution of this work must maintain attribution to the author(s) and the published article's title, journal citation, and DOI. Funded by SCOAP . charged anti-de Sitter (AdS) BHs [4]. In this case, for a certain range of pressures, when temperature is lowered monotonically, a large/small/large BH reentrant phase transition occurred. Further studies show that for higher than four-dimensional BI-AdS BHs, there is no RPT [5]. In [6], $d$-dimensional singly spinning Kerr-AdS BHs were studied and it was shown that RPT appears for $d \geq 6$. Remarkably, in these two BH systems, an RPT is accompanied by a Hawking-Page (HP) phase transition. This fact is interesting and important since it has been shown that the HP phase transition is related to a confinement/deconfinement PT in quark-gluon plasma [7]. More studies on RPT in higherdimensional single-spinning and multispinning Kerr-AdS and Kerr-dS BHs have been carried out in [8-11]. It is worth mentioning that these examples of BH RPTs are accompanied by a jump at the global minimum of the Gibbs free energy. This discontinuity is referred to as zeroth-order PT and seen for instance in superfluidity and superconductivity [12]. Recently, it has been shown that the zeroth-order PT can take place as well in an extended phase space of charged dilaton black holes [13]. RPTs have also been observed in frameworks consisting of higher-curvature corrections [14-18]. In these kinds of gravity theories it is possible to find multiple RPTs, and/or RPTs in which there is no zerothorder PT, with the RPT taking place by a succession of some first order PTs $[14,16,17]$.

In this paper, we explore a possible microscopic origin of the black hole RPT via Ruppeiner geometry $[19,20]$. We 
compare the behaviors of Ricci scalar of Ruppeiner geometry $R$ (referred to as Ruppeiner invariant) for the situations in which an RPT appears and try to infer its microscopic origin. We do this in the case of BI-AdS BHs as well as singly spinning Kerr-AdS BHs. The sign of the Ruppeiner invariant indicates the dominant interaction between possible molecules of a $\mathrm{BH}(R>0$ : repulsion, $R<0$ : attraction and $R=0$ : no interaction) [21-23], while its magnitude is a measure of the average number Planck areas on the event horizon that are correlated with each other [24] (for more information, see [25,26] and references therein). Thermodynamic geometry of anyon gas were also studied in [27]. We note that the microscopic behavior of possible $\mathrm{BH}$ molecules has been previously studied via Ruppeiner geometry [28-31].

Our paper is organized as follows. In Sec. II, we review the subject of RPT in the context of black hole thermodynamics. For this purpose, we shall consider two cases: static AdS black holes in the presence of nonlinear BI electrodynamics and spinning Kerr-AdS black holes. We shall use the terminology SPT (for standard phase transition) to denote any phase transition that is not reentrant (i.e., not RPT), whereas PT shall refer to any possible phase transition without distinction. In Sec. III, we study the Ruppeiner geometry of SPTs and RPTs to understand the microscopic origin of the latter. In Sec. IV, we summarize the results we found in this paper.

\section{REVIEW OF BLACK HOLE RPT}

In this section, we review thermodynamics of higherdimensional BI-AdS BHs as well as singly spinning KerrAdS BHs and discuss the situations under which the RPT appears in these configurations. Our discussions here are based on Refs. [5,6].

\section{A. BI-AdS black holes}

The metric of a $d$-dimensional BI-AdS BH is

$$
d s^{2}=-f(r) d t^{2}+\frac{d r^{2}}{f(r)}+r^{2} d \Omega_{d-2}^{2},
$$

in which $d \Omega_{d-2}^{2}$ is the line element of the $(d-2)$ dimensional hypersurface with constant scalar curvature $(d-2)(d-3)$ and volume $\omega$ and

$$
\begin{aligned}
f(r)= & 1+\frac{r^{2}}{l^{2}}-\frac{m}{r^{d-3}} \\
& +\frac{4 b^{2} r^{2}}{(d-1)(d-2)}\left(1-\sqrt{1+\frac{(d-2)(d-3) q^{2}}{2 b^{2} r^{2 d-4}}}\right) \\
& +\frac{2(d-2) q^{2}}{(d-1) r^{2 d-4}} \\
& \times{ }_{2} F_{1}\left[\frac{d-3}{2 d-4}, \frac{1}{2}, \frac{3 d-7}{2 d-4},-\frac{(d-2)(d-3) q^{2}}{2 b^{2} r^{2 d-4}}\right],
\end{aligned}
$$

where $l$ is the AdS radius of spacetime, $m$ and $q$ are related to total mass and charge of $\mathrm{BH}$ as

$$
Q=\frac{q \omega}{4 \pi} \sqrt{\frac{(d-2)(d-3)}{2}}, \text { and } M=\frac{(d-2) m \omega}{16 \pi},
$$

where $\omega$ represents the area of the unit $(d-2)$-sphere. Using the fact that $f\left(r_{+}\right)=0$ where $r_{+}$is the radius of outermost horizon, we can find the constant $m$ in terms of $r_{+}$and express the total mass of $\mathrm{BH}$ as a function of $r_{+}$,

$$
\begin{aligned}
M= & \frac{(d-2) \omega r_{+}^{d-3}}{16 \pi}\left[1+\frac{r_{+}^{2}}{l^{2}}\right. \\
& +\frac{4 b^{2} r_{+}^{2}}{(d-1)(d-2)}\left(1-\sqrt{1+\frac{16 \pi^{2} Q^{2}}{b^{2} \omega^{2} r_{+}^{2 d-4}}}\right) \\
& +\frac{64 \pi^{2} Q^{2}}{(d-1)(d-3) \omega^{2} r_{+}^{2 d-6}} \\
& \left.\times{ }_{2} F_{1}\left[\frac{d-3}{2 d-4}, \frac{1}{2}, \frac{3 d-7}{2 d-4},-\frac{16 \pi^{2} Q^{2}}{b^{2} \omega^{2} r_{+}^{2 d-4}}\right]\right] .
\end{aligned}
$$

In the above expression $b$ is the nonlinear $\mathrm{BI}$ parameter appears in the BI Lagrangian, $L_{\mathrm{BI}}=$ $4 b^{2}\left(1-\sqrt{1+F^{\mu \nu} F_{\mu \nu} / 2 b^{2}}\right)$. The electrodynamics field tensor $F_{\mu \nu}=\partial_{[\mu} A_{\nu]}$ and gauge potential $A_{\nu}=$ $\left(A_{t}, 0,0, \ldots\right)$ is given by

$$
\begin{aligned}
A_{t}= & -\frac{q}{r^{d-3}} \sqrt{\frac{d-2}{2(d-3)}} \\
& \times{ }_{2} F_{1}\left[\frac{d-3}{2 d-4}, \frac{1}{2}, \frac{3 d-7}{2 d-4},-\frac{(d-2)(d-3) q^{2}}{2 b^{2} r^{2 d-4}}\right] .
\end{aligned}
$$

In the limiting case where $b \rightarrow \infty$, the BI Lagrangian reproduces the linear Maxwell one. The electromagnetic potential defined by

$$
\Phi=\left.A_{\mu} \chi^{\mu}\right|_{r \rightarrow \infty}-\left.A_{\mu} \chi^{\mu}\right|_{r=r_{+}}
$$

where $\chi=\partial_{t}$ is the Killing vector, can also be calculated as

$$
\begin{aligned}
\Phi= & \frac{4 \pi Q}{(d-3) \omega r_{+}^{d-3}} \\
& \times{ }_{2} F_{1}\left[\frac{d-3}{2 d-4}, \frac{1}{2}, \frac{3 d-7}{2 d-4},-\frac{16 \pi^{2} Q^{2}}{b^{2} \omega^{2} r_{+}^{2 d-4}}\right] .
\end{aligned}
$$

The cosmological constant is fixed as $\Lambda=-(d-1)(d-2) / 2 l^{2}$ and related to the thermodynamic pressure as (in Planck unit) [32-35]

$$
P=-\frac{\Lambda}{8 \pi}=\frac{(d-1)(d-2)}{16 \pi l^{2}} .
$$


The corresponding Hawking temperature associated with the event horizon $r_{+}$, and the entropy of $\mathrm{BH}$ are given by

$$
\begin{gathered}
T=\frac{1}{4 \pi}\left[\frac{(d-1) r_{+}}{l^{2}}+\frac{(d-3)}{r_{+}}\right. \\
\left.+\frac{4 b^{2} r_{+}}{d-2}\left(1-\sqrt{1+\frac{16 \pi^{2} Q^{2}}{b^{2} \omega^{2} r_{+}^{2 d-4}}}\right)\right], \\
S=\frac{\omega r_{+}^{d-2}}{4} .
\end{gathered}
$$

Interpreting the mass of $\mathrm{BH}, M$, as the enthalpy rather than the internal energy of the gravitational system [33], we can write the first law of thermodynamics as

$$
d M=T d S+\Phi d Q+V d P+\mathfrak{B} d b,
$$

in which $M$ is a function of $\mathrm{BI}$ coupling coefficient as well as other usual thermodynamic parameters [36]. The conjugate quantity of $b$ is given by

$$
\begin{aligned}
\mathfrak{B}= & \frac{\omega b r_{+}^{d-1}}{2(d-1) \pi}\left(1-\sqrt{1+\frac{16 \pi^{2} Q^{2}}{b^{2} \omega^{2} r_{+}^{2 d-4}}}\right) \\
& +\frac{4 Q^{2} \pi}{(d-1) b \omega r_{+}^{d-3}} \\
& \times{ }_{2} F_{1}\left[\frac{d-3}{2 d-4}, \frac{1}{2}, \frac{3 d-7}{2 d-4},-\frac{16 \pi^{2} Q^{2}}{b^{2} \omega^{2} r_{+}^{2 d-4}}\right],
\end{aligned}
$$

which is referred to as "BI vacuum polarization." Also, the thermodynamic volume conjugate to the pressure is $V=\omega r_{+}^{d-1} /(d-1)$. The generalized Smarr formula in the extended phase space can be obtained by scaling argument as

$$
M=\frac{d-2}{d-3} T S+\Phi Q-\frac{2}{d-3} V P-\frac{1}{d-3} \mathfrak{B} b .
$$

To examine the PT behaviors (or thermodynamic behaviors), in addition to the equation of state $T=T(P, V)$, which can be calculated by eliminating $l^{2}$ between (8) and (9), we study the Gibbs free energy $G=M-T S$,

$$
\begin{aligned}
G(T, P)= & \frac{\omega}{16 \pi}\left[r_{+}^{d-3}-\frac{16 \pi P r_{+}^{d-1}}{(d-1)(d-2)}\right. \\
& -\frac{4 b^{2} r_{+}^{d-1}}{(d-1)(d-2)}\left(1-\sqrt{1+\frac{16 \pi^{2} Q^{2}}{b^{2} \omega^{2} r_{+}^{2 d-4}}}\right) \\
& +\frac{64(d-2)^{2} \pi^{2} Q^{2}}{(d-1)(d-3) \omega^{2} r_{+}^{d-3}} \\
& \left.\times_{2} F_{1}\left[\frac{d-3}{2 d-4}, \frac{1}{2}, \frac{3 d-7}{2 d-4},-\frac{16 \pi^{2} Q^{2}}{b^{2} \omega^{2} r_{+}^{2 d-4}}\right]\right] .
\end{aligned}
$$

Note that in our study on the BI-AdS BH, we treat $Q$ and $b$ as fixed variables.

\section{B. Singly-spinning Kerr-AdS black holes}

In $d$-dimensional spacetime the metric of singly spinning Kerr-AdS black holes may be written

$$
\begin{aligned}
d s^{2}= & -\frac{\Delta}{\rho^{2}}\left(d t-\frac{a}{\Xi} \sin ^{2} \theta d \varphi\right)^{2}+\frac{\rho^{2}}{\Delta} d r^{2}+\frac{\rho^{2}}{\Sigma} d \theta^{2} \\
& +\frac{\Sigma \sin ^{2} \theta}{\rho^{2}}\left[a d t-\frac{\left(r^{2}+a^{2}\right)}{\Xi} d \varphi\right]^{2}+r^{2} \cos ^{2} \theta d \Omega_{d-2}^{2},
\end{aligned}
$$

where

$$
\begin{aligned}
& \Delta=\left(r^{2}+a^{2}\right)\left(1+\frac{r^{2}}{l^{2}}\right)-\frac{2 m}{r^{d-5}}, \\
& \Xi=1-\frac{a^{2}}{l^{2}}, \\
& \Sigma=1-\frac{a^{2}}{l^{2}} \cos ^{2} \theta \text { and } \rho^{2}=r^{2}+a^{2} \cos ^{2} \theta .
\end{aligned}
$$

The associated thermodynamic quantities read (in Planck units)

$$
\begin{gathered}
M=\frac{\omega}{4 \pi} \frac{m}{\Xi^{2}}\left(1+\frac{(d-4) \Xi}{2}\right), \\
J=\frac{\omega}{4 \pi} \frac{m a}{\Xi^{2}}, \\
\Omega_{H}=\frac{a}{l^{2}} \frac{r_{+}^{2}+l^{2}}{r_{+}^{2}+a^{2}}, \\
P=\frac{-\Lambda}{8 \pi}=\frac{(d-1)(d-2)}{16 \pi l^{2}}, \\
T=\frac{1}{2 \pi}\left[\left(1+\frac{r_{+}^{2}}{l^{2}}\right)\left(\frac{r_{+}}{a^{2}+r_{+}^{2}}+\frac{d-3}{2 r_{+}}\right)-\frac{1}{r_{+}}\right], \\
S=\frac{\omega}{4} \frac{\left(a^{2}+r_{+}^{2}\right) r_{+}^{d-4}}{\Xi},
\end{gathered}
$$

where $m$ can be calculated by setting $\Delta\left(r_{+}\right)=0$. We find

$$
m=\left(r_{+}^{2}+a^{2}\right)\left(\frac{r_{+}^{d-5}}{2}+\frac{r_{+}^{d-3}}{2 l^{2}}\right)
$$

One can also find the first law of BH thermodynamics and Smarr formula as [33]

$$
\begin{gathered}
d M=T d S+\Omega_{H} d J+V d P, \\
\frac{d-3}{d-2} M=T S+\Omega_{H} J-\frac{2}{d-2} V P,
\end{gathered}
$$



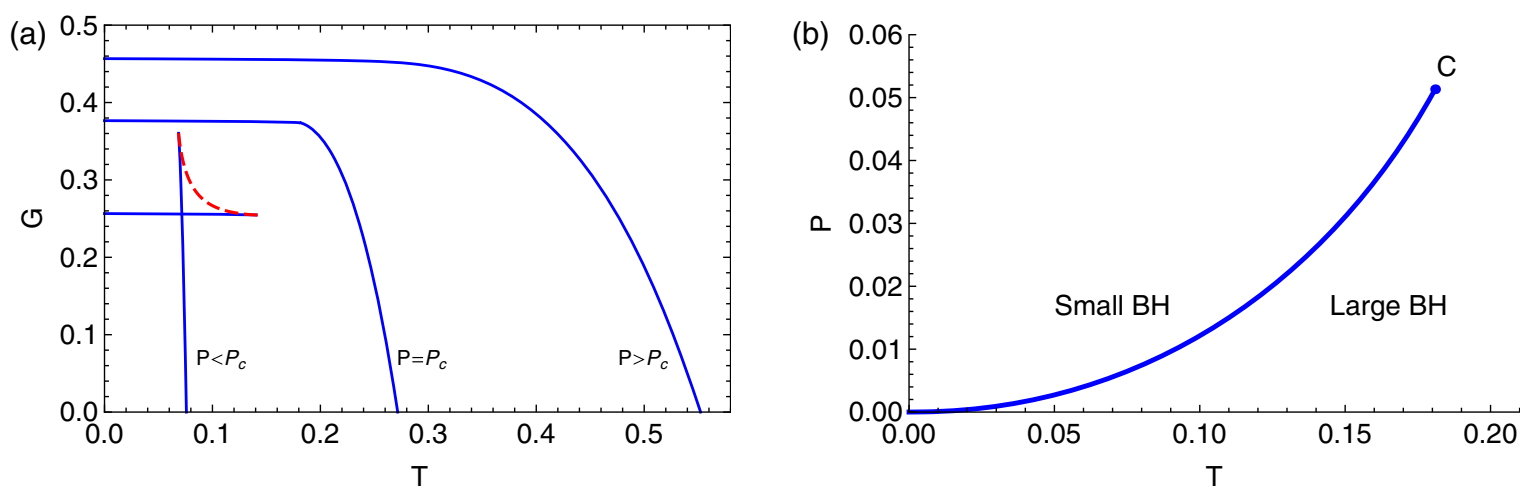

FIG. 1. The behavior of $G$ and $P$ versus $T$ for five-dimensional BI-AdS BHs with $Q=1$ and $b=0.035$ where $\left(P_{c}, T_{c}\right)=(0.051,0.181)$. This behavior is the same for $(n \geq 5)$-dimensional BI-AdS and four- and five-dimensional Kerr-AdS BHs. (a) $G-T$ diagram for different values of $P$. Solid blue and dashed red lines correspond to $C_{P}>0$ and $C_{P}<0$, respectively. (b) $P-T$ diagram. $c$ denotes the critical point.

where the volume conjugate to pressure is

$$
V=\frac{\omega r_{+}^{d-1}}{d-1}\left[1+\frac{a^{2}}{\Xi} \frac{1+r_{+}^{2} / l^{2}}{(d-2) r_{+}^{2}}\right]
$$

The Gibbs free energy $G=M-T S$ governing the thermodynamic behavior of the system reads

$$
\begin{aligned}
G(T, P, J)= & \frac{\omega r_{+}^{d-5}}{16 \pi \Xi^{2}} \\
& \times\left(3 a^{2}+r_{+}^{2}-\frac{\left(r_{+}^{2}-a^{2}\right)^{2}}{l^{2}}+\frac{3 a^{2} r_{+}^{4}+a^{4} r_{+}^{2}}{l^{4}}\right) .
\end{aligned}
$$

Eliminating $m, a$ and $r_{+}$in Eqs. (17)-(22) in terms of the basic thermodynamic variables, one can numerically obtain the equation of state $T=T(P, V)$ in the canonical ensemble (with fixed $J$ ).

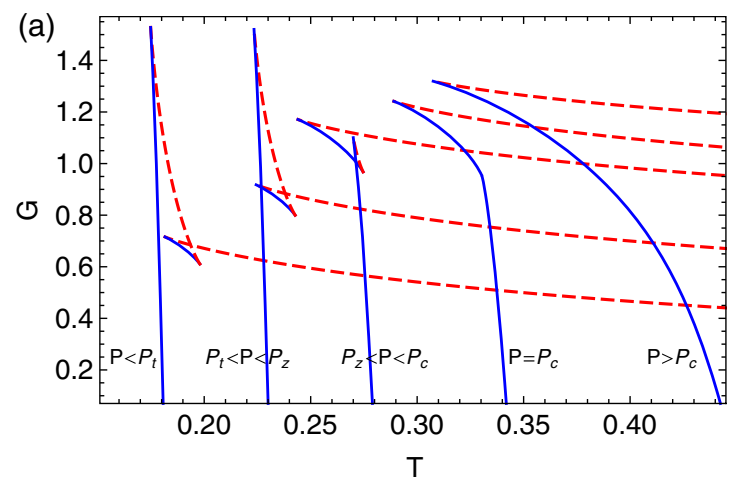

\section{Reentrant phase transition}

The thermal stability of a thermodynamic system may be determined by the sign of the system's response functions. One of theses response functions is specific heat at constant pressure. For the BHs under consideration, the specific heat is given by

$$
\begin{gathered}
C_{P}=T\left(\frac{\partial S}{\partial T}\right)_{P,\{Y\}} \\
\{Y\}= \begin{cases}b, Q & \text { for BI-AdS } \\
J & \text { for Kerr-AdS }\end{cases}
\end{gathered}
$$

Positivity (negativity) of $C_{P}$ determines the local stability (instability) which is shown by solid blue (dashed red) lines in a $G-T$ diagram [see Figs. 1(a) and 2]. In order to examine the precise behavior of the system, we investigate the behavior of the Gibbs free energy $G$. For fivedimensional BI-AdS black holes this is plotted in Fig. 1(a). From this figure, we see that for $P>P_{c}, G$

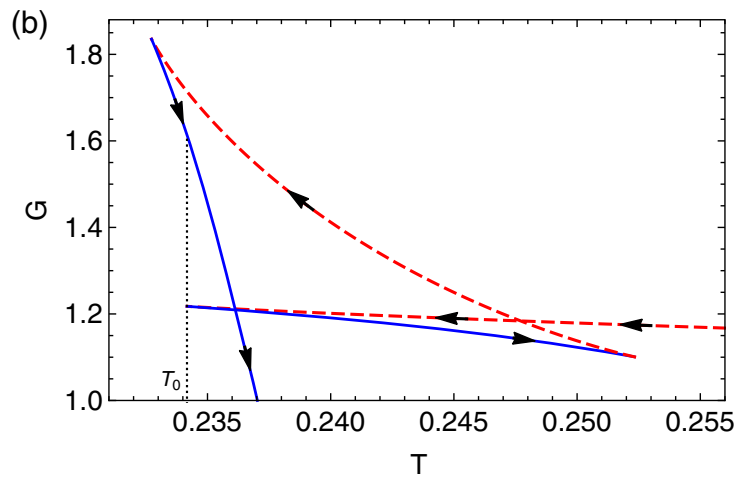

FIG. 2. The behavior of $G$ versus $T$ for the six-dimensional Kerr-AdS black hole with $J=1$. Solid blue and dashed red lines are corresponding respectively to $C_{P}>0$ and $C_{P}<0$. This behavior is qualitatively the same for four-dimensional BI-AdS and $(n \geq 6)$ dimensional Kerr-AdS BHs. (a) The behavior of $G$ for different pressures where $\left(P_{c}, P_{z}, P_{t}\right)=(0.0957,0.0578,0.0553)$ and $\left(T_{c}, T_{z}, T_{t}\right)=(0.3002,0.2349,0.2331)$. Note that various curves are shifted for clarity. (b) The behavior of $G$ for $P=0.0568$. The arrows show the direction of event horizon radius increase. The zeroth-order $P_{T}$ takes place at $T_{0}=0.2335$. 
has smooth behavior as a function of $T$, whereas for $P<P_{c}$ it exhibits multivalued behavior related to the $\mathrm{LBH} / \mathrm{SBH}$ (large black hole/small black hole) first order SPT that is similar to the SPT of the van der Waals fluid system. The corresponding phase diagram is shown in Fig. 1(b) in which for $P<P_{c}$ and $T<T_{c}$, large and small $\mathrm{BH}$ phases are separated by the transition line. This kind of behavior is observed for $(d \geq 5)$-dimensional BI-AdS BHs and also four- and five-dimensional Kerr-AdS BHs.

However for four-dimensional BI-AdS $\mathrm{BHs}$ and $(d \geq 6)$-dimensional Kerr-AdS BHs, the thermodynamic behavior is different. The behavior of the Gibbs free energy for six-dimensional Kerr-AdS BHs versus temperature for fixed pressure is plotted in Fig. 2(a). As one can see, for $P>P_{c}$ the behavior of the system is similar to that of Schwarzschild-AdS BHs: the upper (lower) branch corresponds to small (large) BHs with $C_{P}<0\left(C_{P}>0\right)$ and at $G\left(T_{H P}, P_{H P}\right)=0$, the Hawking-Page SPT occurs. Decreasing the pressure to $P=P_{c}$, we have an additional second order PT at $T=T_{c}$ in comparison with $P>P_{c}$ case. In the region $P_{t}<P<P_{c}$, a LBH/SBH first order SPT takes place which is similar to liquid/gas van der Waals SPT (note that $P_{t}$ shows the end of a transition line corresponding to the first order SPT). In the region $P_{t}<P<P_{z}$, according to the behavior of Gibbs free energy, we have a zeroth-order PT besides the LBH/SBH first order PT [see Fig. 2(b)]. Note that $P_{z}$ determines the starting point for observing zeroth-order PT. This kind of phase transition takes place by a discontinuity in Gibbs free energy that gives rise to a small/large (intermediate) BH PT. Therefore, generally we have a LBH/SBH/LBH phase transition - this is an RPT since the starting and ending phases are macroscopically similar. For $P<P_{t}$ the situation is the same as the $P>P_{c}$ case; note that since the black hole is charge a Hawking-Page PT (which would not conserve charge) does not take place.

The phase diagram corresponding to an RPT is depicted in the $P-T$ diagram in Fig. 3. The solid lines are coexistence lines denoting the boundary of two different phases. The inset shows that for $P>P_{z}$ there is a SPT, whereas for $P<P_{t}$ there is no PT, even where $G$ changes sign. The RPT occurs for $P_{t}<P<P_{z}$, where the red line signifies a first-order PT and the magenta line signifies a zeroth-order PT. Note that there is a forbidden region in parameter space for which no black hole solutions exist. Similar RPT behavior takes place for four-dimensional BIAdS as well as $(d \geq 6)$-dimensional Kerr-AdS BHs [4,6].

For $P_{t}<P=0.0567<P_{z}$ an RPT corresponding to a six-dimensional Kerr-AdS BHs is depicted in Fig. 2(b). The direction of arrows follows increasing horizon radius. As temperature decreases the system always moves on the lower branch in each region to maintain thermodynamic equilibrium. Beginning on the lower steeply sloped curve, a first order PT takes place after which the system is on the near-flat blue curve. This is an SPT from an LBH to

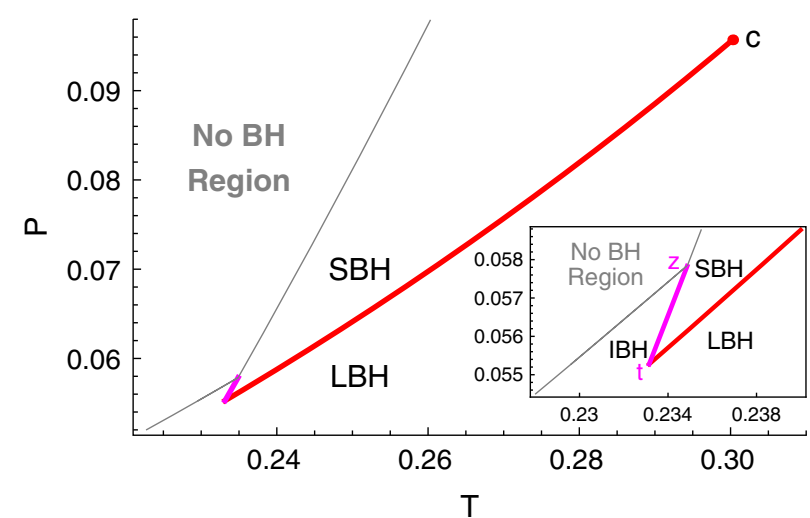

FIG. 3. Phase diagram for the six-dimensional Kerr-AdS BH with $J=1$. $c$ denotes the critical point. We zoom in on the RPT region to make it more clear. "No BH Region" corresponds to a forbidden region of parameter space. This behavior is the same for $(n \geq 6)$-dimensional Kerr-AdS and four-dimensional BI-AdS BHs.

an SBH. At $T=T_{0}$, a zeroth-order PT takes place at which a finite jump in $G$ takes place and the system moves back to the steep blue curve. This finite jump is a zeroth-order PT between an SBH and an IBH (an LBH but of smaller radius). The entire process is an RPT.

\section{MICROSCOPIC ORIGIN OF THE BLACK HOLE RPT}

In this section, we will study the microscopic origin of black holes RPT by adopting the Ruppeiner approach towards thermodynamic geometry of BHs [21-26]. We define the Ruppeiner metric in $X^{\alpha}=(M, Z)$ space where $Z=Q$ for BI-AdS BHs and $Z=P$ for Kerr-AdS BHs. The entropy $S$ leads to the thermodynamic potential,

$$
g_{\alpha \beta}=-\frac{\partial^{2} S}{\partial X^{\alpha} \partial X^{\beta}} .
$$

The above metric can also be rewritten in the Weinhold form

$$
g_{\alpha \beta}=\frac{1}{T} \frac{\partial^{2} M}{\partial Y^{\alpha} \partial Y^{\beta}},
$$

where $Y^{\alpha}=(S, Z)$. The Ricci scalar corresponding to this metric is referred to as Ruppeiner invariant $R$, and can give us some information about the microscopic behavior of possible $\mathrm{BH}$ molecules.

Whereas the classical approach toward BHs is silent with regard to their degrees of freedom, the Ruppeiner geometry provides us with a tool for shedding light on the properties of possible $\mathrm{BH}$ microstructures. Indeed, the Ruppeiner invariant is used to determine the microscopic properties and character of thermodynamic systems [21-26], including the character of microscopic interactions and average 

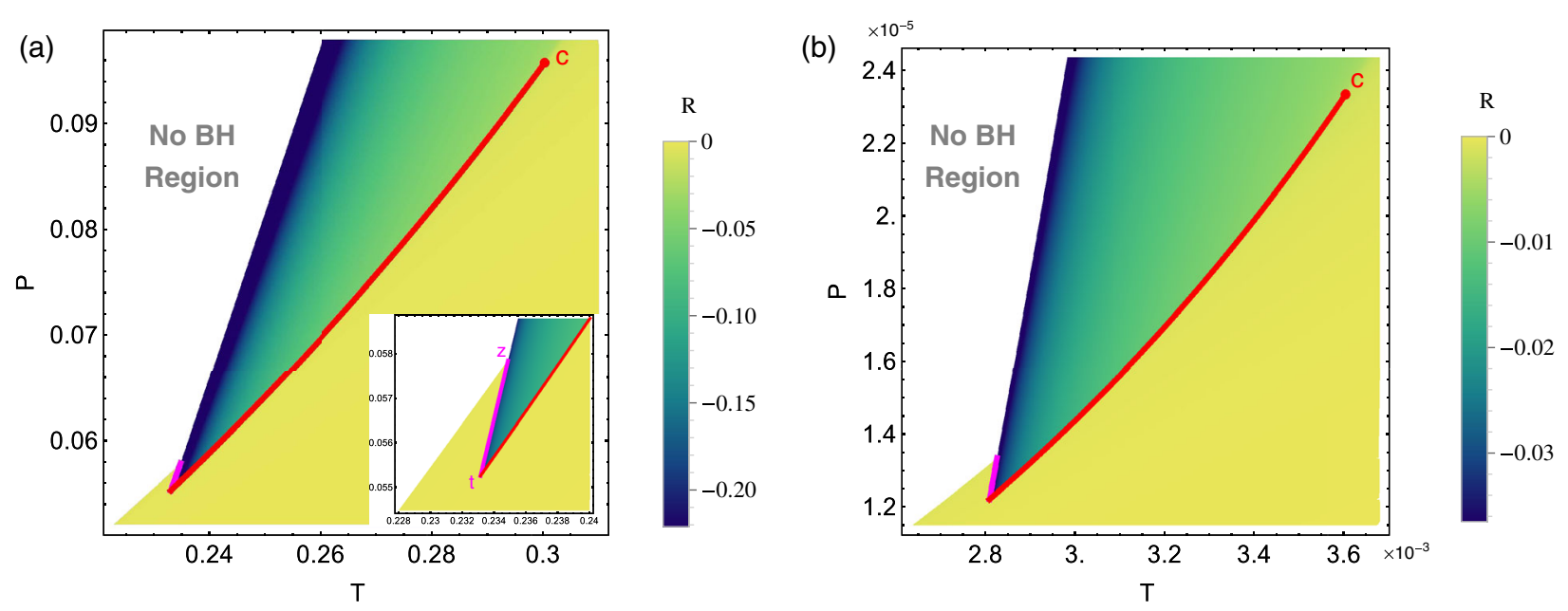

FIG. 4. The $R-P-T$ diagram for four-dimensional BI-AdS and six-dimensional Kerr-AdS BHs. This behavior is the same for $(n \geq 6)$-dimensional Kerr-AdS and four-dimensional BI-AdS BHs. (a) Six-dimensional Kerr-AdS with $J=1$. (b) Four-dimensional BI-AdS with $Q=1$ and $b=0.035$.

size of organized fluctuations. The sign of $R$ signifies the dominant interaction between microscopic constituents of a system (the possible $\mathrm{BH}$ molecules in our case): positive, negative and zero values respectively indicate repulsion, attraction, and no interaction [21-23]. Its magnitude provides a measure of the average number of correlated constituents [24]; for black holes this would be the average number of correlated Planck areas on the event horizon.

In order to study the microscopic origin of an RPT, we examine the behavior of the Ruppeiner invariant on both sides of the coexistence lines in the $P-T$ diagram. We illustrate this in Fig. 4 for both four-dimensional BI-AdS black holes and for six-dimensional singly spinning KerrAdS black holes; note that both diagrams are similar to 3 . Since there are two PTs in case of RPT, we have two transition lines. The rightmost and leftmost transition lines are respectively related to $\mathrm{LBH} / \mathrm{SBH}$ and $\mathrm{SBH} / \mathrm{IBH}$ (a smaller LBH) PTs as temperature is decreased.

We see for both cases that $R<0$, indicating that the dominant interaction is attractive. The important point we can understand from Fig. 4 is that for the zeroth-order PT $R$ is much more negative in going from the SBH to the IBH than in going from the LBH to the SBH. For the RPT, $R$ goes from small negative (LBH) to larger negative (SBH) back to small negative (IBH) again by decreasing the temperature at fixed pressure. According to the definition of RPT, we know that the starting and ending phases should be the same macroscopically. From Fig. 4 we see that they are almost the same too, from a microscopic point of view, since the magnitude and sign of the Ruppeiner invariant $R$ are the same on these phases.

We see from Fig. 4 that when we move downward through the rightmost transition line, the value of $R$ for points close to the line and on its right side (i.e., the LBH phase) is near zero. This behavior can be seen more clearly in Fig. 5. For the usual first-order PT, the magnitude of the Ruppeiner invariant $R$ for the LBH phase near the transition line is almost the same as for the IBH, i.e., it is near zero. For these black holes their microscopic constituents attract and have weak correlation. This behavior is expected for large $\mathrm{BHs}$ where microscopic ingredients of $\mathrm{BH}$ are expected to be far from each other.

However, for the SBH phase, the microscopic behavior of BHs underlying the usual PT and RPTs are different near the transition line. For the underlying RPT, as we move downward near the coexistence line in Fig. 5 on its left side, the Ruppeiner invariant is negative and of increasing magnitude. The dominant interaction is attractive, with the constituents much more strongly correlated.

The behavior of $R$ for the usual SPT in Fig. 1(b) is illustrated in Fig. 6. In this case, the value $R$ near the

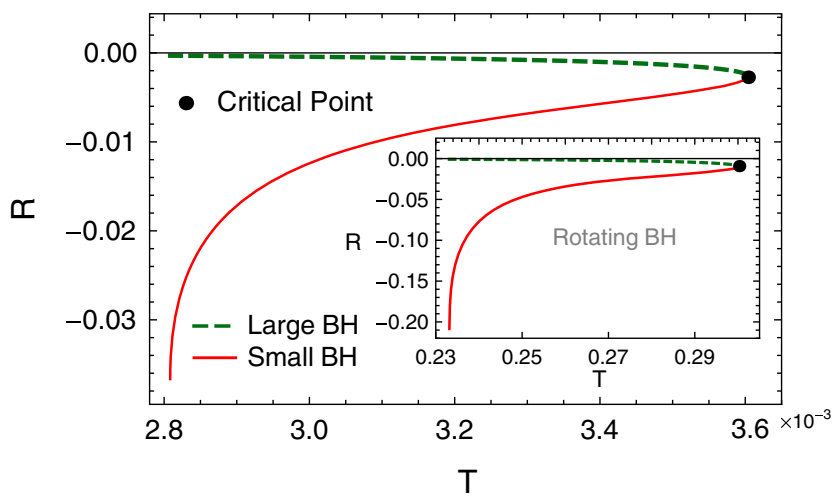

FIG. 5. The behavior of the Ruppeiner invariant $R$ along the rightmost transition line (the red line in Fig. 4) for fourdimensional BI-AdS and six-dimensional Kerr-AdS BHs. For $(n \geq 6)$-dimensional Kerr-AdS BHs, this behavior is qualitatively the same. 


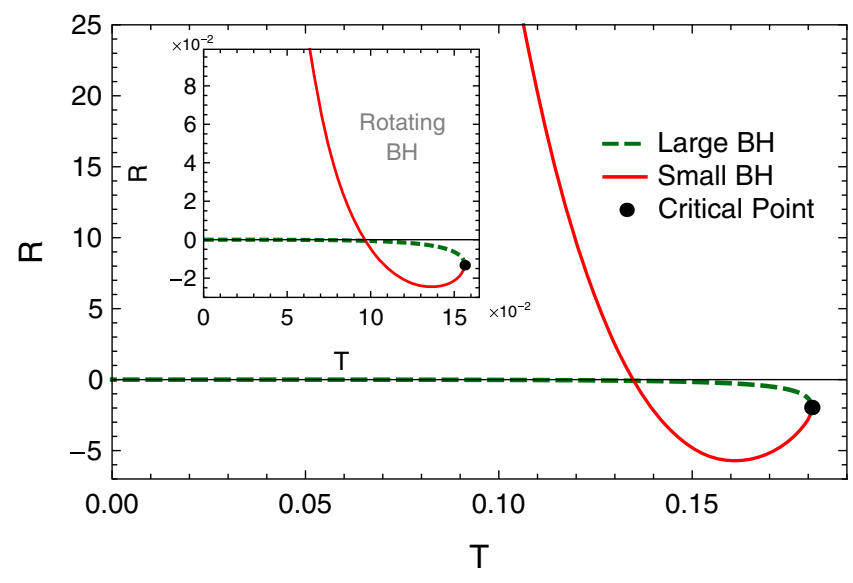

FIG. 6. The behavior of Ruppeiner invariant $R$ along the transition line fore five-dimensional BI-AdS in Fig. 1(b) and Kerr-AdS BHs with $J=1$, with the values of $b$ and $Q$ as in Fig. 1 . For $(n \geq 5)$-dimensional BI-AdS and four- and five-dimensional Kerr-AdS BHs, the behavior is qualitatively the same.

coexistence line for the SBH is negative at the critical point but passes through zero toward increasingly positive values (signifying increasing correlation for repulsively interacting constituents) as the temperature decreases; the value of $R$ is positive infinite near zero temperature. We know that for SBHs near the transition line, the pressure is low when temperature is low, as is clear from Fig. 1(b). In this region of the phase diagram for the SBH the repulsion is high; despite this, the pressure is low. Evidently the effects of low temperature dominate over this repulsion, "freezing" the black hole molecules and increasing their correlation. The LBH always has weak attraction and low correlation amongst its constituents.

It is worth noting that the RPT occurs in a special region of low pressures $P_{t}<P<P_{z}$, shown in Fig. 4. For a given pressure in this region, by decreasing the temperature we transit from an $\mathrm{LBH}$ to an $\mathrm{SBH}$ of greater $|R|$, indicating greater correlations in comparison to other ranges of pressure. This high correlation becomes higher as we decrease the temperature further in this pressure range. One of the signs of a PT is a change from low to high correlation amongst constituents (or vice versa). It seems that from a microscopic point of view, the difference making the BH system able to undergo an RPT for this special region of pressure $P_{t}<P<P_{z}$ is the greater correlation between molecules of SBHs in comparison to other ranges of pressure.

Here, a question may arise when one compares the behavior of the Ruppeiner invariant $R$ for $\mathrm{BH}$ configurations exhibiting an RPT and ones exhibiting an SPT. For the latter, the correlation (the magnitude of $R$ ) is high for small BHs near the transition line for low pressures as one can see from Fig. 6 [note from Fig. 1(b) that along transition line low temperatures lead to low pressures]. Furthermore, as one can see in Fig. 7, for a given (low) pressure, when the

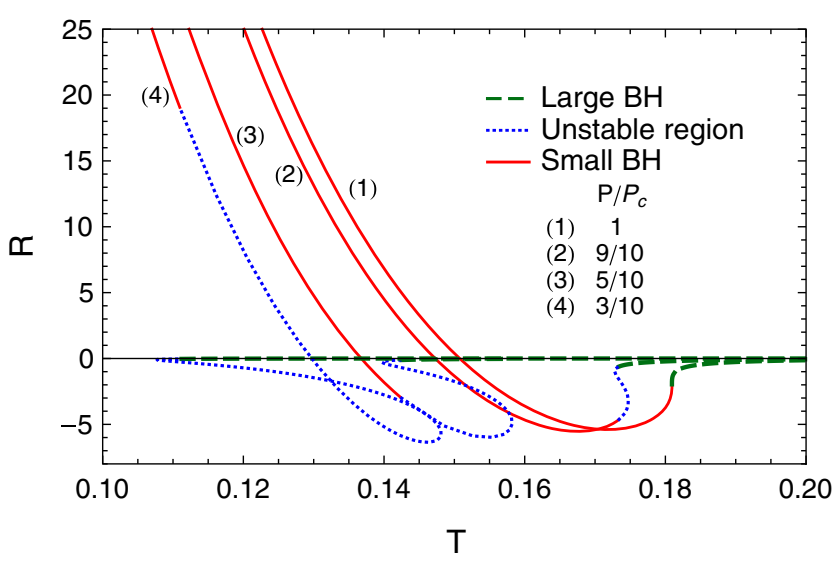

FIG. 7. The behavior of Ruppeiner invariant $R$ for different given pressures for five-dimensional BI-AdS BHs with $Q=1$ and $b=0.035$. For other configurations underlying usual PT, the behavior is the same.

temperature decreases and system transits to an SBH phase, the correlation (the magnitude of $R$ ) increases as temperature decreases more in this phase. The SPT/RPT distinction is evidently one of increasing repulsion/attraction for an $\mathrm{SBH}$ relative to the $\mathrm{LBH}$. The attraction may help the SBH to undergo an additional zero-order PT as temperature decreases (hence an RPT) whereas the increasing repulsion obstructs an additional phase transition (hence an SPT).

As one can see in Fig. 7, the BH configuration with SPT shows two different behaviors by decreasing the temperature from high to low values for a given pressure. A part of this behavior for high temperatures carries the bosonic negative sign whereas another part for low temperatures looks like a fermionic gas. Similar behavior has been observed for a quantum gas of anyons where the molecular volume is fixed [26,27,37]. The blue dotted lines in Fig. 7 correspond to the swallowtail part of the $G-T$ diagram for $P<P_{c}$ [Fig. 1(a)]. In the swallowtail region, the system has a thermodynamic instability on part of the curve, whereas on the other part the specific heat $C_{P}$ at fixed $P$ (which determines the local instability) is positive.

\section{SUMMARY AND CONCLUSIONS}

In this paper, we investigated the microscopic origin of the reentrant phase transition (RPT) in BH thermodynamics. For this purpose, we employed the Ruppeiner geometry towards thermodynamics geometry of BHs. We showed that the ending and starting phases which are the same macroscopically, are the same microscopically, too. We found out that there is no microscopic difference between the behavior of large BHs near the large-small transition line of usual PT and RPT cases, and the main difference is in the microscopic behavior of small black holes. We observed that for usual PTs, the dominant interaction for small BHs near the transition line for low temperatures is repulsive whereas for RPT it is attractive. 
The fact that we have two phase transitions in the RPT case, for the pressure in the range of $P_{t}<P<P_{z}$, can be understood easily [see Fig. 2(a)]. Indeed, in this region the system transits from a large $\mathrm{BH}$ to a small one with higher correlations compared to the other regions of pressure. We showed that with decreasing the temperature of a given pressure $P$, correlation becomes higher and it makes the system possible to underlie another phase transition in the region $P_{t}<P<P_{z}$.

We also explored the microscopic origin of black hole RPTs by adopting the Ruppeiner approach towards thermodynamic geometry of BHs. It is well known that the magnitude of the Ruppeiner invariant $R$ measures the average number of Planck areas on the event horizon correlated to each other or roughly speaking correlation [24]. We examined the behavior of the Ruppeiner invariant on both sides of transition lines in the $P-T$ diagram (see Fig. 4). We observed that in the RPT case the small BHs behave like a bosonic gas whereas in the usual PT case small black holes behave like a quantum anyon gas [27,37]. We showed that the freezing is more effective than the dominant interaction at low temperature since in both the two kinds of phase transitions (namely the usual PT and RPT) have low pressure in this region despite different interactions.

\section{ACKNOWLEDGMENTS}

We are grateful to Professor Bin Wang for useful discussions and helpful comments. M. K.Z. would like to thank Shahid Chamran University of Ahvaz, Iran for supporting this work. A. D. and A. S. thank the research council of Shiraz University. The work of M. K. Z. has been supported financially by Research Institute for Astronomy \& Astrophysics of Maragha (RIAAM) under Research Project No. 1/5237-57. R. B. M. thanks the Natural Sciences and Engineering Research Council of Canada for partial support.
[1] C. Hudson, Die gegenseitige Loslichkeit von Nikotin in Wasser, Z. Phys. Chem. 47, 113 (1904).

[2] O. Panella and P. Roy, Reentrant phase transitions in noncommutative quantum mechanics, J. Phys. Conf. Ser. 670, 012040 (2016).

[3] T. Narayanan and A. Kumar, Reentrant phase transitions in multicomponent liquid mixtures, Phys. Rep. 249, 135 (1994).

[4] S. Gunasekaran, R. B. Mann, and D. Kubiznak, Extended phase space thermodynamics for charged and rotating black holes and Born-Infeld vacuum polarization, J. High Energy Phys. 11 (2012) 110.

[5] D. C. Zou, S. J. Zhang, and B. Wang, Critical behavior of Born-Infeld AdS black holes in the extended phase space thermodynamics, Phys. Rev. D 89, 044002 (2014).

[6] N. Altamirano, D. Kubiznak, and R. B. Mann, Reentrant phase transitions in rotating anti-de Sitter black holes, Phys. Rev. D 88, 101502 (2013).

[7] E. Witten, Anti-de Sitter space, thermal phase transition, and confinement in gauge theories, Adv. Theor. Math. Phys. 2, 505 (1998).

[8] N. Altamirano, D. Kubiznak, R. B. Mann, and Z. Sherkatghanad, Kerr-AdS analogue of triple point and solid/liquid/gas phase transition, Classical Quantum Gravity 31, 042001 (2014).

[9] N. Altamirano, D. Kubiznak, R. B. Mann, and Z. Sherkatghanad, Thermodynamics of rotating black holes and black rings: Phase transitions and thermodynamic volume, Galaxies 2, 89 (2014).

[10] D. Kubiznak and F. Simovic, Thermodynamics of horizons: De Sitter black holes and reentrant phase transitions, Classical Quantum Gravity 33, 245001 (2016).
[11] S. W. Wei, P. Cheng, and Y.X. Liu, Analytical and exact critical phenomena of $d$-dimensional singly spinning Kerr-AdS black holes, Phys. Rev. D 93, 084015 (2016).

[12] V. P. Maslov, Zeroth-order phase transitions, Mathematical notes of the Academy of Sciences of the USSR 76, 697 (2004).

[13] A. Dehyadegari, A. Sheykhi, and A. Montakhab, Novel phase transition in charged dilaton black holes, Phys. Rev. D 96, 084012 (2017).

[14] A. M. Frassino, D. Kubiznak, R. B. Mann, and F. Simovic, Multiple reentrant phase transitions and triple points in Lovelock thermodynamics, J. High Energy Phys. 09 (2014) 080.

[15] S. W. Wei and Y. X. Liu, Triple points and phase diagrams in the extended phase space of charged Gauss-Bonnet black holes in AdS space, Phys. Rev. D 90, 044057 (2014).

[16] R. A. Hennigar, W. G. Brenna, and R. B. Mann, Pv criticality in quasitopological gravity, J. High Energy Phys. 07 (2015) 077.

[17] Z. Sherkatghanad, B. Mirza, Z. Mirzaeyan, and S. A. H. Mansoori, Critical behaviors and phase transitions of black holes in higher order gravities and extended phase spaces, Int. J. Mod. Phys. D 26, 1750017 (2017).

[18] R. A. Hennigar and R. B. Mann, Reentrant phase transitions and van der Waals behavior for hairy black holes, Entropy 17, 8056 (2015).

[19] G. Ruppeiner, Thermodynamics: A Riemannian geometric model, Phys. Rev. A 20, 1608 (1979).

[20] G. Ruppeiner, Riemannian geometry in thermodynamic fluctuation theory, Rev. Mod. Phys. 67, 605 (1995); Erratum, Rev. Mod. Phys. 68, 313(E) (1996). 
[21] G. Ruppeiner, Thermodynamic curvature measures interactions, Am. J. Phys. 78, 1170 (2010).

[22] G. Ruppeiner, Thermodynamic curvature from the critical point to the triple point, Phys. Rev. E 86, 021130 (2012).

[23] H. O. May, P. Mausbach, and G. Ruppeiner, Thermodynamic curvature for attractive and repulsive intermolecular forces, Phys. Rev. E 88, 032123 (2013).

[24] G. Ruppeiner, Thermodynamic curvature and phase transitions in Kerr-Newman black holes, Phys. Rev. D 78, 024016 (2008).

[25] G. Ruppeiner, Thermodynamic curvature: Pure fluids to black holes, J. Phys. Conf. Ser. 410, 012138 (2013).

[26] G. Ruppeiner, Thermodynamic curvature and black holes, Springer Proc. Phys. 153, 179 (2014).

[27] B. Mirza and H. Mohammadzadeh, Nonperturbative thermodynamic geometry of anyon gas, Phys. Rev. E 80, 011132 (2009).

[28] S. W. Wei and Y.X. Liu, Insight into the Microscopic Structure of an AdS Black Hole from Thermodynamical Phase Transition, Phys. Rev. Lett. 115, 111302 (2015); Erratum, Phys. Rev. Lett. 116, 169903(E) (2016).

[29] M. Kord Zangeneh, A. Dehyadegari, and A. Sheykhi, Comment on "Insight into the microscopic structure of an AdS black hole from a thermodynamical phase transition", arXiv:1602.03711.
[30] A. Dehyadegari, A. Sheykhi, and A. Montakhab, Critical behavior and microscopic structure of charged AdS black holes via an alternative phase space, Phys. Lett. B 768, 235 (2017).

[31] M. Kord Zangeneh, A. Dehyadegari, M. R. Mehdizadeh, B. Wang, and A. Sheykhi, Thermodynamics, phase transitions and Ruppeiner geometry for Einstein-dilaton Lifshitz black holes in the presence of Maxwell and Born-Infeld electrodynamics, Eur. Phys. J. C 77, 423 (2017).

[32] M. M. Caldarelli, G. Cognola, and D. Klemm, Thermodynamics of Kerr-Newman-AdS black holes and conformal field theories, Classical Quantum Gravity 17, 399 (2000).

[33] D. Kastor, S. Ray, and J. Traschen, Enthalpy and the mechanics of AdS black holes, Classical Quantum Gravity 26, 195011 (2009).

[34] M. Cvetic, G. Gibbons, D. Kubiznak, and C. Pope, Black hole enthalpy and an entropy inequality for the thermodynamic volume, Phys. Rev. D 84, 024037 (2011).

[35] B. P. Dolan, Where is the PdV term in the first law of black hole thermodynamics?, arXiv:1209.1272.

[36] S. Gunasekaran, R. B. Mann, and D. Kubiznak, Extended phase space thermodynamics for charged and rotating black holes and Born-Infeld vacuum polarization, J. High Energy Phys. 11 (2012) 110.

[37] B. Mirza and H. Mohammadzadeh, Ruppeiner geometry of anyon gas, Phys. Rev. E 78, 021127 (2008). 